Instituto Internacional de Investigación y Desarrollo Tecnológico Educativo INDTEC, C.A.

DOI: https://doi.org/10.29394/Scientific.issn.2542-2987.2021.6.21.16.301-313

OAI-PMH: http://www.indteca.com/ojs/index.php/Revista Scientific/oai

Ensayo Original / Original Essay

\title{
Planificación Educativa desde el Imaginario Docente en la Educación Básica
}

Autora: Juana Maribel Reyes Galicia Universidad Pedagógica Experimental Libertador, UPEL reyesgaliciaabc@gmail.com Puerto Cabello, Venezuela https://orcid.org/0000-0003-2421-6079

\section{Resumen}

La planificación educativa del imaginario docente en los últimos años ha incidido considerablemente en el proceso de la enseñanza y el aprendizaje de los estudiantes, principalmente en la forma como el educador ha abordado dicho proceso a través de las planificaciones diarias y la construcción de los proyectos de aprendizajes, esquivando las técnicas, estrategias y recursos como elementos fundamentales en la planeación vistas en las reformas curriculares así como también en el currículo del sistema educativo bolivariano, por consiguiente a través de este ensayo se pretende comprender el significado que le atribuye el imaginario para alcanzar una planificación que brinde respuestas a las necesidades de los estudiantes donde se refleje la contextualización de su entorno social, porque existe el docente seguro en la elaboración de las mismas consiguiendo así la transformación en la educación. Este se fundamenta en las Teorías de los Imaginarios Sociales de Pintos (1995); el Imaginario Docente de Castoriadis (1975); y la Teoría del interaccionismo simbólico de Blumer (1969). El mismo se enmarcó en el enfoque fenomenológico hermenéutico lo que permitió el acercamiento a la comprensión del entorno social de estos imaginarios docentes en la praxis diaria desde, de su diario vivir.

Palabras clave: planificación educativa; imaginario docente; significados.

Código de clasificación internacional: 5802.04 - Niveles y temas de educación.

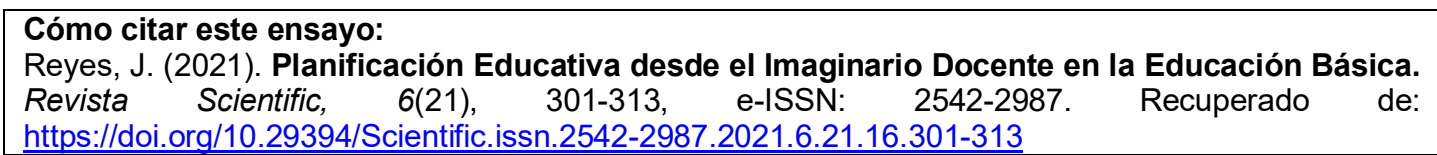

Fecha de Recepción: 01-03-2021
Fecha de Aceptación: 29-06-2021
Fecha de Publicación: 05-08-2021 


\title{
Educational Planning from the Teacher Imaginary in Basic Education
}

\begin{abstract}
The educational planning of the teaching imaginary in recent years has had a considerable impact on the teaching and learning process of students, mainly in the way the educator has approached this process through daily planning and the construction of teaching projects. learning, avoiding the techniques, strategies and resources as fundamental elements in the planning seen in the curricular reforms as well as in the curriculum of the Bolivarian educational system, therefore through this essay it is intended to understand the meaning attributed by the imaginary to achieve a planning that provides responses to the needs of students where the contextualization of their social environment is reflected, because the teacher is sure in the elaboration of them, thus achieving the transformation in education. This is based on the Theories of Social Imaginaries by Pintos (1995); the Imaginary Docent of Castoriadis (1975); and Blumer's Theory of Symbolic Interactionism (1969). It was framed in the hermeneutical phenomenological approach, which allowed the approach to the understanding of the social environment of these imaginary teachers in the daily praxis from their daily lives.
\end{abstract}

Keywords: educational planning; teaching imaginary; meanings. International classification code: 5802.04 - Levels and subjects of education.

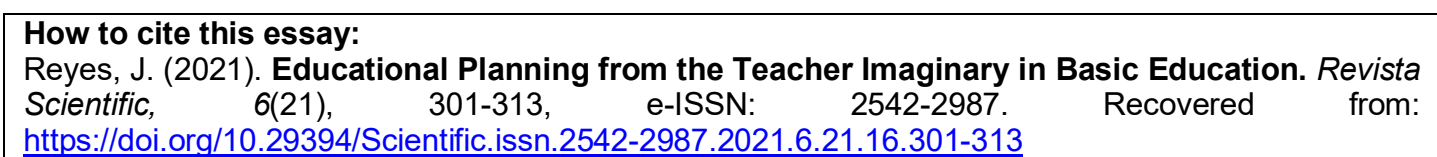

Date Received:

01-03-2021
Date Acceptance:

29-06-2021
Date Publication: 05-08-2021 


\section{Introducción}

La planificación ha hecho posible que los individuos conozcan ante las necesidades y adversidades como organizar su forma de vida y también su relación en los diferentes contextos en la cual se desenvuelven, es decir el hombre que proyecta sus acciones le permite tener un mayor control sobre ciertos planes que se establece basados en objetivos o metas ,que se pueden lograr a través de un periodo bien sea a corto, mediano o a largo plazo, por consiguiente la vida misma, y el quehacer diario del hombre conllevan que cada actividad sea planificada, esto permite el desenvolvimiento en el entorno y en la sociedad. En este sentido Robbins y Coulter (2005), expresan que:

La planeación consiste en definir las metas de la organización, establecer una estrategia general para alcanzarlas y trazar planes exhaustivos para integrar y coordinar el trabajo de la organización. La planeación se ocupa tanto de los fines (qué hay que hacer) como de los medios (cómo hay que hacerlo) (pág. 158).

Entonces se podría decir que la planificación permite organizar el sistema de trabajo dentro de una organización con el fin de mejorar o transformar una realidad. Por lo que a través de la planificación los imaginarios docentes asumen un compromiso de crear los proyectos de acuerdo a las exigencias de las reformas educativas, tratando de organizarse en la búsqueda de técnicas y estrategias que consoliden los fines de la educación ,así como también los pilares como es el aprender a ser, aprender a convivir, aprender hacer y aprender a reflexionar, apoyándose en los propósitos para la transformación social del individuo en una sociedad cambiante, considerando que la planificación educativa va de la mano con las políticas educativas de nuestro país, creadas por el Ministerio del Popular para la Educación y los organismos internacionales como la Unesco entre otros.

Dichas políticas educativas son las que forjan las estrategias, proyectos, planes y programas escolares en la educación, por lo que es necesario realizar 
un diagnóstico social para determinar cuáles acciones son las adecuadas y darle respuesta al sistema escolar, con el fin de determinar que dichas políticas sean captadas y asumidas en cada institución educativa, es decir las políticas educativas surgen para completar todo ese sistema de organización a nivel institucional que es necesario.

Por ejemplo, en Venezuela las políticas están determinadas por los programas transversalizados del currículo es decir, se encuentran fuera del currículo tales como el Programa Todas las Manos a la Siembra, la Valija Didáctica, el Plan de Lectura Revolucionario, el Proyecto Canaima, el Plan de Ahorro Energético, entre otros, los cuales permite que los docentes puedan trabajar, sin embargo estos planes, proyectos y programas en su mayoría resulta dificultoso en el desarrollo o inclusión transversal dentro de la planificación.

A pesar de ese compromiso asumido por algunos imaginarios docentes en cuanto a la planificación educativa, en el subsistema de la Educación Básica, perteneciente al Estado Falcón se ha podido visualizar mediante una vista a dichas instituciones que los imaginarios docentes no están posesionados en utilizar la planificación educativa como herramienta en su labor escolar.

Aun cuando se han realizado adecuaciones curriculares y formaciones docentes seguimos observando inseguridad o dudas en la elaboración o construcción de las planificaciones, evidenciándose desinterés en realizarla, lo que conlleva en la copia y pega de las planificaciones de otros imaginarios docentes fuera del contexto real lo que trae como consecuencias dificultad para adaptarlas a un grupo de estudiantes para la cual no fue elaborada ni planificada en los proyectos de aprendizajes y por consiguiente en las planificaciones diarias.

El objetivo principal es comprender, entender e interpretar cómo, actúan los imaginarios docentes en el desarrollo de las planificaciones que se 
llevan a cabo en los ambientes de clases, a fin de fortalecer el proceso de la enseñanza y el aprendizaje teniendo como centro a los estudiantes de igual manera los aportes que surgen en las representaciones mentales de cada educador, puesto que la planificación es un proceso que debe realizarse de manera sistemática, equilibrada y argumentada para la población de discente que se incorporan en el sistema educativo. Seguidamente dejar al descubierto que la planificación es necesaria en cualquier contexto y sobre todo en el educativo que tiene como meta la transformación del hombre nuevo y el alcance en la calidad de la educación.

\section{Desarrollo}

A medida que ha transcurrido el tiempo la planificación ha ido tomando más impulso en las diferentes organizaciones sobre todo en el ámbito educativo, se ha convertido en la principal herramienta con lo que cuenta el imaginario docente para desarrollar el trabajo administrativo y la planificación en el aula.

En este mismo sentido, la evolución trae como consecuencia adaptarse a las realidades, el hombre en busca de ir perfeccionando el desenvolvimiento en las organizaciones ha tenido que ir desarrollando nuevas maneras de planificar y con ella los métodos utilizados para su elaboración y cumplir de esta forma con los planes y objetivos trazados. De aquí surge la importancia que tiene la planificación en todos los contextos de elaborar los planes pertinentes respetando cada fase en cada una de las instituciones de manera de lograr una planificación eficiente y eficaz.

Se deduce entonces que los docentes en su imaginario deben establecer las metas para lograr cumplir de forma satisfactoria los objetivos planteados al momento de elaborar las planificaciones basándose principalmente en los fundamentos filosóficos y psicológicos curriculares de la educación. En cuanto al fundamento filosófico, Sequiera (1994a): plantea que: 
Lo filosófico ayuda a orientar la vida, a saber quiénes somos, por qué somos como somos y hacia dónde vamos. Lo filosófico tiene que ver con nuestra forma de vida, nuestros problemas y proyectos $y$, sobre todo, con nuestra organización de pensamiento, decisiones, gustos, escogencias y alternativas (pág. 43).

Este fundamento tiene que ver con el ser, es decir la persona el ser humano en sí, permite entonces que el imaginario docente le de valor a los proyectos y por ende las planificaciones curriculares. Por otro lado, Sequiera (1994b): también contempla "[...] la fundamentación psicológica del currículo tiene que ver con los conceptos que proporciona la psicología en relación con los procesos del aprendizaje [...]" (pág. 45). Dicho de otro modo, el imaginario docente debe ir enfocado en el conocimiento, motivación y características del estudiantado con el fin de que le permita apoderarse del conocimiento, es necesario para ello que cada imaginario docente pueda elaborar planificaciones que permitan adquirir dichos conocimientos con facilidad y efectividad que den repuestas en el proceso de enseñanza.

Ahora bien, cada estudiante comprende y participa en el proceso educativo de diferentes formas, en este sentido el imaginario docente debe tomar en cuenta además de la participación de estos en la construcción de ese conocimiento, las individualidades de cada estudiante, así como las planificaciones grupales con el fin de adecuar las estrategias en función de cada uno de los educandos tomando como punto de referencia el contexto donde se ubican.

En este contexto existe una serie de aspectos que deben considerarse cuando se planifica entre ellos se menciona, para quien se planifica, las necesidades e interés del grupo, la población estudiantil que determina la toma de decisiones de acuerdo a su nivel de competencias alcanzadas y el contexto, el uso de herramientas para el aprendizaje, la implementación de técnicas como juegos, dinámicas, canciones, entre otros, de igual manera considerar 
las características percibidas por los niños de su entorno familiar y social bien sea objetiva o de forma subjetiva acompañada de las experiencias, de modo que la planificación sea flexible para todos.

Entonces es necesario la puesta en práctica de estos elementos en la elaboración de las planificaciones, que responda en lograr el aprendizaje y la restructuración del currículo para adquirir los conocimientos que fortalezcan la transformación de los procesos pedagógicos.

\subsection{Teoría de los Imaginarios Sociales de Pintos (1995)}

Existen varios referentes teóricos que nos permite comprender el imaginario social referido principalmente a las ciencias sociales, el docente como parte de esa sociedad y como persona nata no se escapa de la concepción como imaginario.

El autor Pintos (1995): nos brinda un gran aporte sobre lo que es los imaginarios en cuanto a la realidad como situación al enfrentarse al mundo social, donde existen colectivos o representaciones sociales, es decir, el objeto y el sujeto acompañado de una serie de significaciones imaginarias al respecto de la temática abordada. Es necesario mencionar los aportes principalmente de Parsons (1937), citado por Pintos (1995), el cual señalan que:

Las representaciones colectivas no son, ellas mismas, la realidad social. Son representaciones de ella. [...] Pero ¿dónde está la "realidad" correspondiente a la que se refieren las representaciones colectivas? Sólo observamos sus "manifestaciones", [...] pero no observamos la "cosa misma" (pág. 9).

Conforme a lo planteado, se desconoce la realidad que habita en cada una de estas representaciones y colectivos sociales, partiendo de su acervo cultural y lo histórico social.

Desde esta perspectiva, las representaciones o colectivos sociales que conforman los imaginarios, son una terminología que no se ha entendido en la 
sociedad profesional quizás por la manera como se ha desarrollado en la actualidad el concepto que se tiene sobre el mismo, tal vez la causa sea el desconocimiento del concepto, las herramientas la praxis y el escaso uso de este tema en los trabajos de investigación social.

\subsection{Teoría del Imaginario Docente de Castoriadis (1975)}

El imaginario social de Castoriadis (1975): constituye una referencia o modo de interpretar la realidad social que debe ser establecida por la sociedad, donde existe la socialización, que activa el mundo de la percepción que garantiza como yo veo el mundo y lo percibo desde esa realidad, dicho de otro modo, la imaginación es algo abstraído de nuestras mentes en un espacio y tiempo determinado que nos permite llegar a un objetivo.

Incluso está estrechamente vinculado a la imaginación y conectada a la experiencia de cada individuo con todo lo que esto conlleva en las creencias, emociones, sentimientos, valores y por supuesto educación. Para este teórico la imaginación radical es la contraposición a la imaginación reproductiva porque emergen nuevas cosas que permite la transformación de esa imaginación.

La imaginación radical es parte de la creación que tiene que ver con la psique o mente producto del efecto de representaciones emocionales que dan pie al conmuevo y a las aspiraciones, pero tal vez esta sea la razón de porque los docentes tienen un gran compromiso y responsabilidad, puesto que de algún modo recae en ellos el bienestar de la sociedad, y es por eso deben poseer un conocimiento amplio, sobre todo tener dominio sobre el arte de enseñar, garantizando así el aprendizaje, al mismo tiempo que forman a los individuos como buenos ciudadanos.

\subsection{Teoría del Interaccionismo Simbólico Blumer (1969)}

Esta es otra de las teorías seleccionadas para el estudio como es el 
interaccionismo simbólico de Blumer (1969): el cual contiene tres elementos básicos fundamentales, en primer lugar, explica que las personas interactúan de acuerdo a la importancia que le dan a los significados producidos por el entorno en el segundo lugar se debe a la interacción que se tiene con las demás personas, es decir cómo se comportan ante los demás y en último lugar se puede decir que estos significados no se mantienen, no son constantes, solo se modifican a medida que las personas van enfrentando nuevas realidades o prácticas.

Para ello, cada sociedad debe ser estudiada según la particularidad de sus miembros. La humanidad es el producto de la interacción con el otro en ese intercambio de creencias, valores, costumbres entre los factores donde interactuamos.

En este intercambio de interacción con los demás, se pone en relieve otros procesos que tienen que ver con la formación del individuo en la sociedad, donde se interpreta a las demás personas que piensan diferente y se comportan de otra forma algunas veces de forma inconsciente y otras veces de forma racional.

Es notable que esta teoría tiene un gran significado en el presente estudio, del imaginario docente ante la elaboración de la planificación, pues permite comprender el significado que esto representa para el docente, teniendo en cuenta que ese significado debe ser usado al momento de plasmar la planificación de las cosas o de los objetos que son importante para que el estudiante se motive y demuestre interés en el aprender.

De igual manera es relevante mencionar la relación que presenta la temática con lo fenomenológico hermenéutico donde es necesario lograr un acercamiento a la comprensión del entorno social, a partir, de la indagación sobre la intersubjetividad narrada por los informantes claves en la planificación educativa. 


\section{Conclusiones}

La planificación educativa es elemental en todo los aspectos administrativos que un docente debe cumplir, por lo que es necesario que tomen en cuenta lo importante que es esta herramienta, para desarrollar el plan diario de clase, también es significativo que los entes encargados revisen, evalúen y le den seguimientos a los planes, programas, proyectos, circulares, reformas educativas, así como también el currículo entre otros, con la intención de que el docente interprete que sin planificación no hay organización, ya que esta permite encontrar respuesta y reestructurar aquellos aspectos que afectan a la educación pero que a través de la actualización se pueden cambiar o mejorar.

Tal es el caso del currículo de la educación Bolivariana que data desde el año 1997 y hasta la presente fecha no se ha actualizado y desde este entonces han ocurrido cambios de paradigmas que en algunos casos han tenido que trabajarse a través de circulares o de temas transversalizados ocasionado por los cambios que ocurren en el sistema educativo en el transcurso del tiempo, que ennoblece en primer lugar la formación de los docentes determinada por la innovación, centrada en la didáctica de la enseñanza y del aprendizaje.

Es por ello que los docentes deben formarse constantemente, capacitarse, y además actualizarse, para que surja el interés y la motivación de cómo desarrollar racionalmente la planificación en la práctica pedagógica, ya que la puesta en práctica desde diferentes escenarios escolares es evidencia que el proceso de la planificación se presenta de manera confusa.

Por lo que se considera debe existir una guía o un modelo que oriente el desarrollo estructural de la planificación, aunque sea un proceso sistemático también racional. Es conveniente que el organismo rector, el Ministerio del Poder Popular para la Educación (MPPE), revise detenidamente estos procesos y actuaciones de los docentes en cuanto a la construcción de la 
planificación, de igual manera el cómo se está desarrollando los contenidos, que técnicas, estrategias se están empleando para que el proceso educativo puede fluir y mejorar la calidad de la educación.

Otro caso relevante conclusivo se debe a la gran influencia que tienen hoy en día las tecnologías de la información y la comunicación, así como también la telemática, se está creando una nueva cibercultura en la educación lo que está generando un cambio en la cultura de los niños.

Esto quiere decir que la planificación está cambiando y debido a esto los docentes deben proyectarse y estar conectado con los nuevo, pues los estudiantes y profesores está siendo afectados en primer lugar porque los docentes de las instituciones públicas no cuentan con estas herramientas para hacerle frente a las planificaciones y en segundo lugar el escaso conocimiento en el manejo de las tecnologías sobre todo del computador y el internet.

Ocasionando que en estas instituciones escolares donde no cuentan con los recursos tecnológicos influye de manera negativa en el proceso de enseñanza y aprendizaje para alcanzar las competencias en esta área y por consiguiente para la realización de las actividades escolares.

En relación con esto es relevante la implementación de estas áreas tecnológicas en los programas de pregrado, post grado, maestría y doctorado para que los docentes logren adquirir estas competencias entre la teoría y la práctica para que de esta manera se logre alcanzar definitivamente la calidad de la educación y se logren los objetivos y los perfiles de los estudiantes contemplados en el sistema educativo Bolivariano.

\section{Referencias}

Blumer. H. (1969). Symbolic Interaction: Perspective and Method. Englewood Cliffs N.J., United States: Prentice Hall. Castoriadis, C. (1975). La institución imaginaria de la sociedad. Volumen

1, Marxismo y teoría revolucionaria. Barcelona, España: Tusquets 
Editores.

Pintos, J. (1995). Los Imaginarios Sociales: La nueva Construcción de la realidad Social. ISBN: 84-293-1151-3. España: Editorial Sal Terrae; Fe y Secularidad.

Robbins, S., \& Coulter, M. (2005). Administración. Octava edición, ISBN: 970-26-0555-5. México: Pearson Educación.

Sequiera A. (1994a,b). Fundamentamentos filosóficos y psicológicos de la planificación curricular. Revista Educación, 18(2), 41-47, e-ISSN: 2215-2644. Recuperado de:

https://doi.org/10.15517/revedu.v18i2.12678 


\section{Ensayo Original / Original Essay}

\section{Juana Maribel Reyes Galicia \\ e-mail: reyesgaliciaabc@gmail.com}

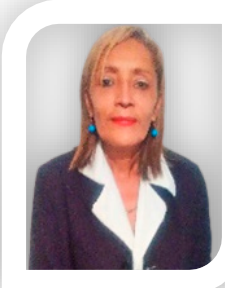

Nacida en Coro, estado Falcón, Venezuela, el 8 de diciembre del año 1968. Licenciada en Educación, egresada de la Universidad Bolivariana de Venezuela (BV); Especialista en Planificación y Evaluación de la Educación por la Universidad Santa María (USM); Magister en Ciencias de la Educación Mención Administración Educativa, titulo obtenido en esta casa de estudio, Universidad Nacional Abierta (UNA); en mi experiencia Laboral Universitaria, fui Facilitadora de la Universidad Bolivariana de Venezuela (UBV); Jurado de diversas investigaciones en trabajos de grado; actualmente Docente de Aula en la Escuela Primaria Bolivariana Próspero Agustín Ocando, Boca de Aroa, estado Falcon, Venezuela.

El contenido de este manuscrito se difunde bajo una Licencia de Creative Commons ReconocimientoNoComercial-Compartirlgual 4.0 Internacional 